\title{
Control of Chloroplast Senescence of Detached Elodea Leaves*
}

\author{
by Yoshio YoshIDA**
}

\author{
Received July 14, 1969
}

\begin{abstract}
Changes in chlorophyll and free sugar contents of Elodea leaves after detachment, and effects of nicotinamide adenine dinucleotide (NAD) and benzimidazole on the changes were investigated. NAD accelerated the loss of chlorophyll, but benzimidazole counteracted the NAD effect and maintained chlorophyll at a high level. Light was found to be necessary for the NAD acceleration of the senescence. Detachment of leaves caused an increase in the free sugar content, while treatment of the detached leaves with NAD caused a decrease. This effect of NAD was also inhibited by benzimidazole.
\end{abstract}

When Elodea leaves are detached and incubated in water, chloroplasts become chlorotic and degenerate. The author ${ }^{1)}$ has shown that the senescence of the chloroplast is closely connected to the activity of the nucleus, and that nicotinamide adenine dinucleotide (NAD) is involved. It has also been reported ${ }^{2)}$ that the senescence of detached wheat leaves could be delayed by treatment with benzimidazole for an extended period after detachment. In our previous work ${ }^{3)}$, electron microscope studies have been carried out to clarify the change of fine structure of chloroplasts in relation to the effects of NAD and benzimidazole on the senescence of Elodea leaves.

The purpose of the present communication is to determine the changes of chlorophyll and sugar contents in connection with the effects of NAD and benzimidazole on the chloroplast senescence.

\section{Materials and Methods}

Elodea densa Casp. was used as an experimental material. They were grown in Hoagland's nutrient solution diluted 20 fold, under illumination with fluorescent lamps for $12 \mathrm{hrs}$. a day and aeration. Leaves from healthy grown tips were excised and rinsed in distilled water. Lots of 300 leaves were immersed in $40 \mathrm{~m} l$ of distilled water, or aqueous solutions of NAD and benzimidazole in final concentrations of $10^{-3} \mathrm{M}$ in petri dishes. Leaf samples taken from one verticilate node were always distrbiuted uniformly into each test solution. The petri dishes were placed in an incubator at about $25^{\circ}$ under continuous illumination with fluorescent lamps $(1500$ lux at the plant level) or in the dark. The process of chloroplast senescence in each medium was followed under a light microscope.

The analysis of pigments was carried out with thin layer chromatography. One

* A part of this work has been done while the author was Professional Associate at the Department of Botany, University of Manitoba, Winnipeg, Manitoba, Canada, 1967-1968.

** Department of Biology, Faculty of Science, Niigata University, Niigata, Japan, 
$\mathrm{g}$ fresh weight of each test sample was previously heated in hot water at $80^{\circ}$ for 3 min. in order to inhibit chlorophyllase activity, then it was ground in a mortar with $10 \mathrm{ml}$ of a mixture of equal volumes of acetone, ethanol, and petroleum ether. After removing sediments by centrifugation at $400 \times \mathrm{g}$ for $10 \mathrm{~min}$., $20 \mu \mathrm{l}$ of the extract was spotted on a silica gel $G$ thin layer plate, and finally developed with the solvent of benzene: acetone $(7: 3)$ mixture. Chlorophyll was extracted from $200 \mathrm{mg}$ fresh weight of each preheated sample by shaking in $10 \mathrm{~m} l$ of $80 \%$ aqueous aceton for 2 hrs. The optical density of the two fold diluted solution of the extract was measured with a photoelectric colorimeter.

Sugar content was determined by "Anthrone Method". One g fresh weight of each test sample was macerated in a mortar with two successive $25 \mathrm{ml}$ aliquots of $70 \%$ ethanol, and the homogenates were centrifuged at $150 \times \mathrm{g}$ for $15 \mathrm{~min}$. The sediment was reextracted with $25 \mathrm{~m} l$ ethanol. The pooled supernatants were evaporated to $5 \mathrm{~m} l$ at $50^{\circ}$ under reduced pressure, then the condensed extract was shaken for $1 \mathrm{hr}$. at $25^{\circ}$ with $1 \mathrm{~g}$ each of ion exchange resins, Dowex $21 \mathrm{~K}$ and Dowex $50 \mathrm{~W}-\mathrm{X} 8$, and left still over-night at $3^{\circ}$. The resins were filtered off and the filtrate was mixed well with $1 \mathrm{~g}$ of talcum powder for $1 \mathrm{hr}$. Chlorophyll free extract was obtained after filtration. $0.5 \mathrm{~m} l$ ice cold sample was dropped carefully along the test tube wall in $5 \mathrm{~m} l$ ice cold anthrone reagent [0.2\% anthrone solution in concentrated sulphuric acid $\left(300 \mathrm{~m} l \mathrm{H}_{2} \mathrm{SO}_{4}\right.$ in $100 \mathrm{~m} l$ water $\left.)\right]$. After mixing by shaking, the test tube was heated in boiling water for $10 \mathrm{~min}$., cooled with running water, and then the absorbancy at $610 \mathrm{~m} \mu$ was estimated. A value of glucose solution $(100 \mu \mathrm{g} / \mathrm{m} l)$ was employed as a standard.

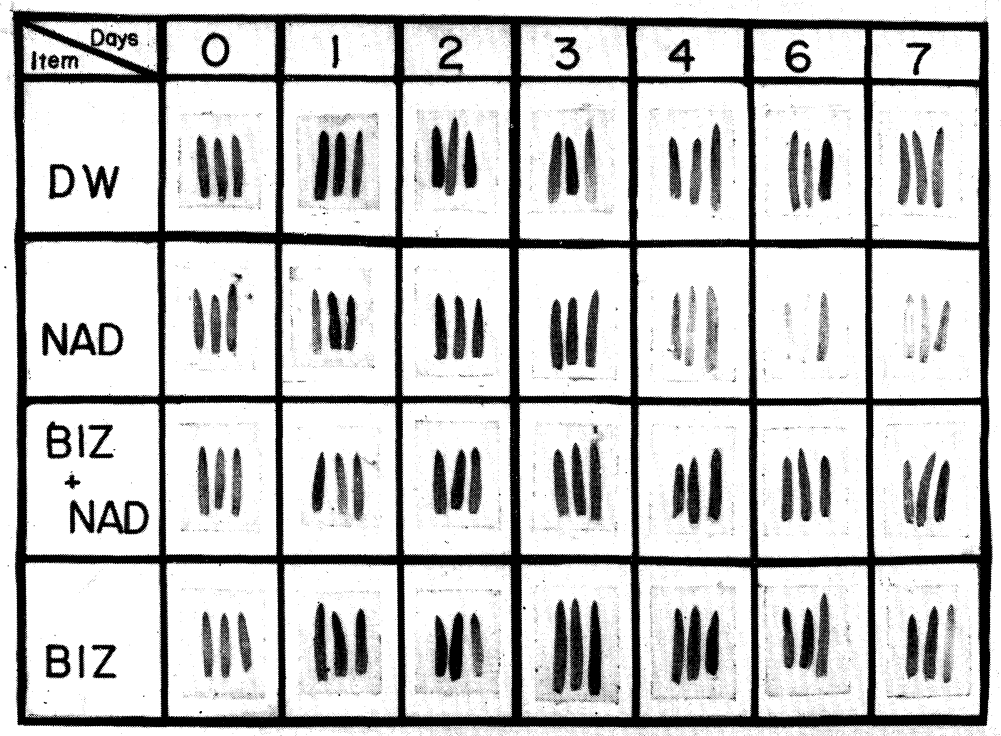

Fig. 1, Representative samples showing the pattern of alteration of Elodea leaves with different treatments after detachment.

DW: Incubated in distilled water.

NAD: Incubation in nicotinamide adnine dinucleotide solution.

BIZ: Incubation in benzimidazole solution. 


\section{Results}

Fig. 1 shows the pattern of alteration of detached Elodea leaves after different treatments during a period of 7 days' incubation. Leaves treated with distilled water gradually lost their green appearance and turned yellow. NAD treatment accelerated the senescence, causing entire chlorosis and deterioration of the leaves. But simultaneous application of benzimidazole with NAD clearly hindered the senescence. Moreover, the leaves treated with benzimidazole only remained relatively deep green during the incubation period, whereas the leaves in water showed remarkable chlorosis after 4 to 5 days. These changes in the leaves were directly derived from the alterations in the chloroplasts of leaf cells, as shown in Fig. 2a-e. NAD markedly accelerated the chloroplast senescence, the chlorosis and shrinking which occurred in distilled water, but benzimidazole clearly overcame the effect of NAD and chloroplasts showed a little deterioration. Moreover, benzimidazole significantly delayed the onset of chloroplast senescence, and the green colour was considerably stabilized and the longevity was extended. These colour and conformational changes of chloroplast should be related with chloroplast function. Indeed, the chloroplasts of the leaves treated with benzimidazole showed an intense reactivity on an iodide test, which was even stronger than that of immediately detached ones, whereas no reactivity was detected in leaves in distilled water or NAD solution (Fig. 3).

The analysis of pigments by thin layer chromatography and comparison of the chlorophyll
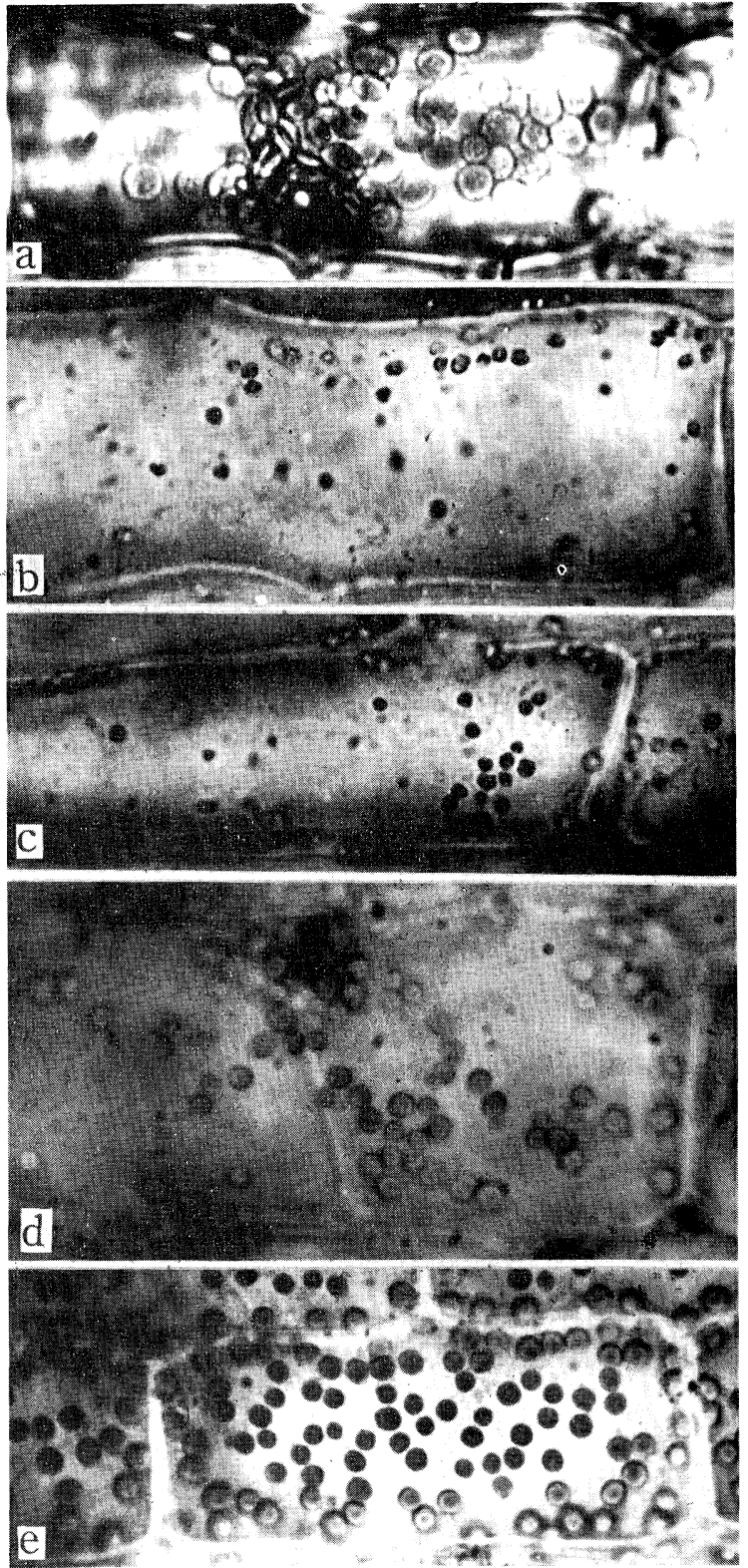

Fig. 2. Photomicrographs showing the chloroplast senescence in leaf cells in each treatment after 6 days incubation.

a: Immediately after detachement.

b: In distilled water.

c: In NAD solution.

$\mathrm{d}$ : In NAD plus benzimidazole solution.

e: In benzimidazole solution. 

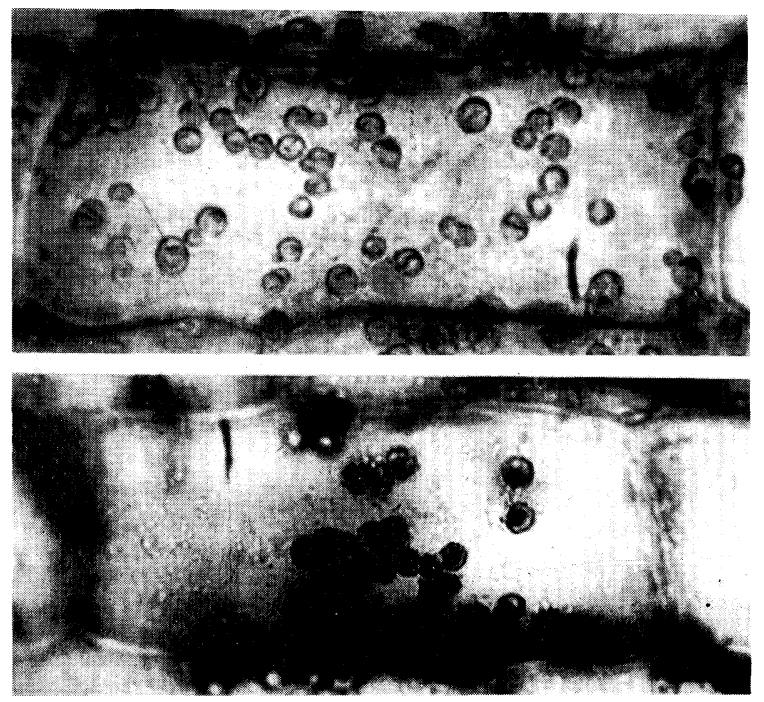

Fig. 3. Iodide stained cells showing the starch content in chloroplasts. Upper: A cell of leaf immediately after detachment.

Lower: Benzimidazole treated one, after 6 days.

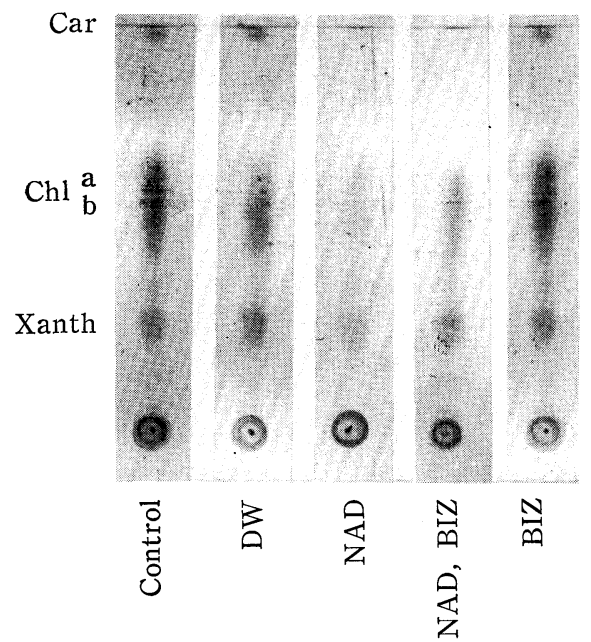

Fig. 4. Thin layer chromatograms showing the chloroplast pigments extracted from leaves after 6 days' incubation in respective solutions.

Car: Carotene.

Chl a: Chlorophyll a.

Chl b: Chlorophyll b.

Xanth: Xanthophyll.

Control: from leaves immediately after detachment.

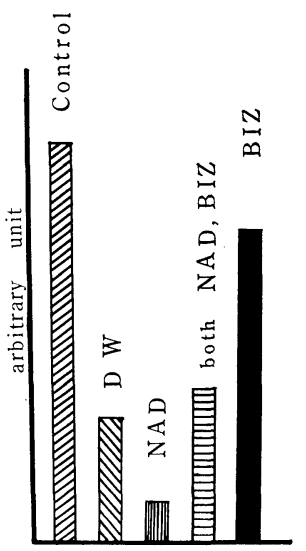

Fig. 5. Comparison of chlorophyll contents of leaves after 6 days in respective solutions. 
depletion in each treatment after 6 days' incubation have shown a pattern almost parallel to the changes in appearance and conformation revealed by microscopic observation. Carotene and xanthophyll showed scarcely any difference in all treatments (Fig. 4 ), and the most striking feature of senescence is a rapid loss of chlorophyll, which is persistent in the visual changes of leaves and chloroplasts in each treatment (Fig. 4, 5). Benzimidazole apparently maintained the chlorophyll content at a high level not much less than that of the control, and clearly overcame the effect of NAD, whereas the leaves incubated in distilled water showed a remarkable decrease in chlorophyll content and NAD accentuated this tendency.

All the incubation experiments described above were done under illumination. When the materials were incubated in the dark, the situation was somewhat different. In general the longevity of the materials was prolonged. Senescence was delayed in all the treatments. Only distilled water treated leaves showed symptoms of etiolation after 15 days of incubation (Fig. 6). Fig. 7 shows time courses of the decrease in relative chlorophyll content of the detached leaves incubated in respective solutions in light or in darkness, which are expressed as percent of that of the control. The leaves kept in continuous darkness survived longer than 30 days, and the chlorophyll contents remained at a high level not only in benzimidazole solution but also in NAD solution even after more than 20 days of incubation. It is clear that light is a necessary

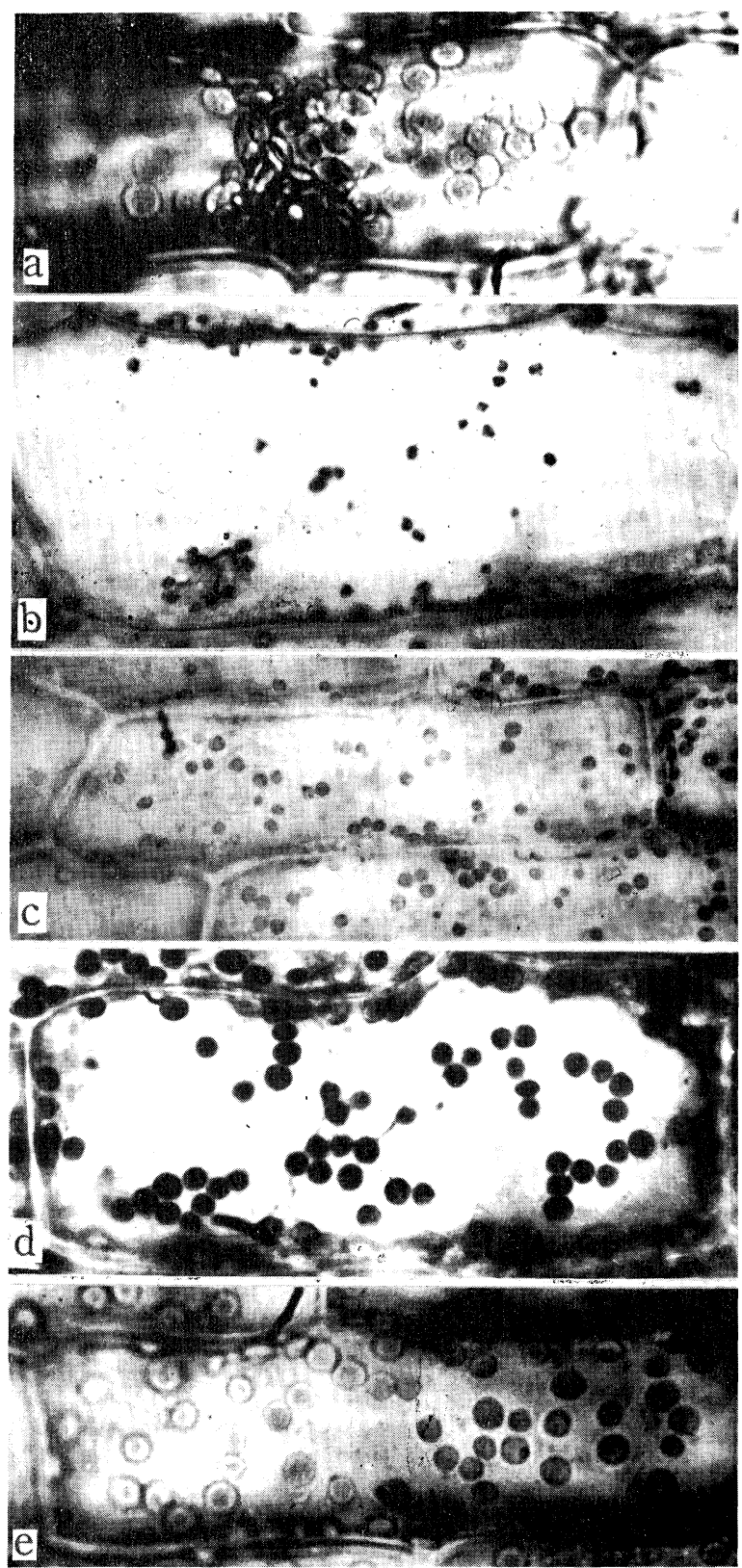

Fig. 6. Photomicrographs showing the chloroplast senescence after 15 days' incubation under dark condition.

a: Immediately after detachment.

$\mathrm{b}$ : In distilled water.

c: In NAD solution.

d: In NAD plus benzimidazole solution.

e: In benzimidazole solution. 


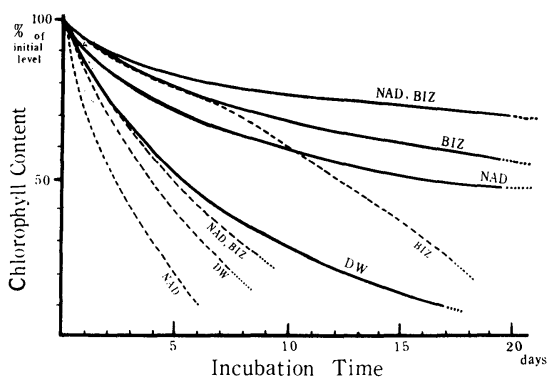

Fig. 7. Time courses of the decrease in relative chlorophyl lcontent of the leaves incubated in different solutions.

Broken lines: Light incubation. Solid lines: Dark incubation.

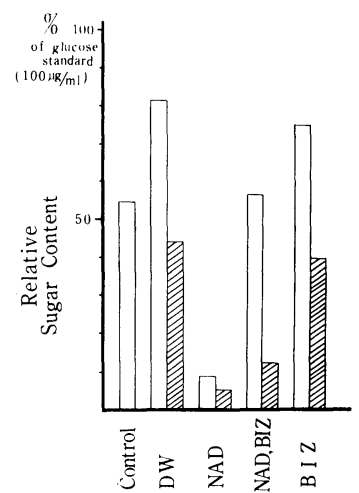

Fig. 8. Column diagram showing changes in relative sugar content of the leaves after 9 days' incubation in respective solutions.

White column: Light incubation. Shadowed column: Dark incubation. factor for NAD-induced senescence of chloroplasts. Especially, it is noteworthy that in darkness NAD effect was reversed, and its simultaneous application rather accentuated the benzimidazole effect of maintaining high chlorophyll content, as shown in Fig. 7.

The changes in sugar content of the leaves after 9 days' incubation in respective solutions are shown in Fig. 8. An accumulation of free sugar over the control was observed in the leaves incubated in distilled water under illumination, and the sugar level was maintained considerably high even in a dark incubation. A marked decrease in sugar content was accompanied by NAD treatment both in the light and in darkness, but this effect of NAD was again counteracted by benzimidazole. However benzimidazole itself did not cause any conspicuous effect on the sugar content as compared with that of distilled water treatment. Since photosynthesis never occurs in the dark, the sugar contents of leaves were lower in darkness than in the light in all cases, but a general tendency was almost the same in both conditions.

\section{Discussion}

It is natural that detached leaves in distilled water show, sooner or later, a chlorotic senescence. The present study showed that NAD drastically accelerated this senescent tendency, and that benzimidazole delayed the onset of senescence and extended the longevity of detached Elodea leaves. The most striking feature of chloroplast senescence was the rapid loss of chlorophyll, whereas the breakdown of other plastid pigments was less pronounced. NAD remarkably enhanced the chlorophyll loss, but benzimidazole efficiently postponed the chlorophyll breakdown. In a previous paper ${ }^{3)}$, it has been reported that the acceleration of leaf senescence by NAD and the retardation by benzimidazole are closely associated with the change of chloroplast fine structure. The maintenance of chlorophyll content at a high level and of the chloroplast fine structure in the presence of benzimidazole is in accordance with the observation that benzimidazole results in the effective maintenance of photophosphorylation of detached wheat leaves at a high level ${ }^{4,5}$. 
The yellowing of detached leaves can temporarily be retarded in darkness. In the dark incubation of detached leaves, it is especially noticeable that NAD also retarded the degradation of chlorophyll to a considerable degree, and that it cooperatively enhanced the benzimidazole effect in maintaining chlorophyll level rather than accelerating its breakdown. No new chlorophyll synthesis occurs in darkness, therefore this result strongly indicates that light is necessary in order for the NAD to accelerate the senescence and the chlorophyll breakdown. This point suggests further complications depending on some photochemical reaction with the mechanism of NAD action on chloroplast senescence.

In detached wheat leaves, senescence is accompanied by a depletion of its reserved carbohydrate and by the increase in free sugar content ${ }^{6)}$. It seems reasonable to assume that a similar situation may exist in Elodea leaves too, as shown in Fig. 8. The extreme decrease of sugar content in leaves treated with NAD may be explained by the fact that NAD is known to be an indispensable coenzyme in a pathway of carbohydrate breakdown and related metobolism. One of the effects of benzimidazole may be conceived to be a depression of NAD action. Indeed Person et $a l^{2)}$ demonstrated that benzimidazole prevented the respiratory increase that occurs after leaf detachment.

The present findings cannot be readily explained, but further investigations of NAD and benzimidazole effects on chloroplast senescence may bring us a due to the understanding of mechanism of maintenance of a proper endogenous balance of chloroplast.

The author wishes to express his thanks to Miss Mioko Ooshio and Mr. Hiroshi Hasegawa for their assistance in a part of this work. The author is also indebted to Dr. E. R. Waygood, head, Department of Botany, University of Manitoba, Winnipeg, Manitoba, Canada, for his encouragement which made this work complete.

\section{References}

1) Yoshida, Y., Protoplasma $54: 476$ (1961).

2) Person, C. D., Sanborski, D. J. and Foryth, F. R., Nature 180: 1294 (1957).

3) Yoshida, Y., Waygood, E. R. and Isaac, P. K., Bot. Mag. Tokyo 82: 424 (1969).

4) Pathak, S. M., PhD. Thesis, Univ. Manitoba,
Canada (1966).

5) Waygood, E. R., Plant Physiol. 40: 1242 (1965).

6) Wang, D. and Hao, M.S. H., Ibid. (Suppl.) 35, XV. (1960). 